\title{
ENTROPY OF RANDOM WALKS ON GROUPS AND THE MACAEV NORM
}

\author{
D. VOICULESCU
}

(Communicated by Jonathan M. Rosenberg)

\begin{abstract}
We show that a discrete group on which there is a finitary random walk with positive entropy satisfies a certain condition involving the Macaev norm. This links the entropy of random walks on groups to the author's work on quasicentral approximate units relative to normed ideals in perturbation theory. On the other hand, the condition we are considering is also an analogue for the Macaev norm of Yamasaki's hyperbolicity condition.
\end{abstract}

\section{INTRODUCTION}

In this paper we provide a necessary condition, involving the Macaev norm, for a finitary random walk on a discrete group to have positive entropy. The entropy of a random walk on a group studied in $[1,2,3,7,11,14]$ is related to the Poisson boundary $[8,9]$. The condition involving the Macaev norm appeared in our work on perturbations of Hilbert space operators $[15,16,17]$. The result we obtain here is similar to the connection we exhibited in [17] between the entropy of a dynamical system and perturbations of operators. Note also the recent appearance of the Macaev ideal in noncommutative differential geometry [6] and of the entropy of a random walk on a group in the theory of subfactors $[13,5]$.

On the other hand the condition involving the Macaev norm can be viewed as the analogue of Yamasaki's $p$-hyperbolicity condition [19] with the $p$-norm replaced by the Macaev norm. As our work in perturbation theory shows, for sharp results in this kind of questions, the $p$-norms or the Orlicz norms considered in [12] should be replaced by the Lorentz $(p, 1)$-norms, of which the Macaev norm is the $(\infty, 1)$-norm.

Given a set $X$ we denote by $\left(l_{\infty}^{-}(X),||_{\infty}^{-}\right)$the Banach space of real functions $f: X \rightarrow \mathbb{R}$ vanishing at infinity, with respect to the norm

$$
|f|_{\infty}^{-}=\sum_{k \in \mathbb{N}} f^{*}(k) k^{-1}
$$

Received by the editors July 5, 1991 and, in revised form, March 3, 1992.

1991 Mathematics Subject Classification. Primary 22D40; Secondary 28D20, 47D50, 47B06, 47B10, 60B15, 60J15.

Research supported in part by a grant from the National Science Foundation and by the Fonds FCAR at CRM, Université de Montréal, where the author was visiting in the spring of 1991. 
where $f^{*}(1) \geq f^{*}(2) \geq \cdots$ is the decreasing rearrangement of the values of $|f|$. The space $l_{\infty}^{-}(X)$ coincides with the Lorentz space $l_{\infty, 1}(X)$. We will often refer to the norm ||$_{\infty}^{-}$as the Macaev norm, since the corresponding normed ideal of compact operators $\mathscr{C}_{\infty}^{-}$is called the Macaev ideal [10]. We will explain at the end of the paper the connection with our work on perturbations.

\section{MAIN RESUlT}

Throughout $G$ denotes a discrete group, $K \subset G$ a finite subset generating $G$, and $\mu$ a probability measure on $G$ with $\operatorname{supp} \mu=K$. We consider the corresponding right random walk on $G$ starting at the neutral element $e \in G$ and with transition probability from $g$ to $h$ given by $\mu\left(g^{-1} h\right)$. By

$$
h(G, \mu)=\lim _{n \rightarrow \infty} n^{-1} H\left(\mu^{* n}\right)
$$

we denote the entropy of the random walk ( $\mu^{* n}$ is the $n$-fold convolution of $\mu$ with itself).

Theorem 1. If $h(G, \mu)>0$, then

$$
\inf \left\{\max _{g \in K}|\alpha(g) f-f|_{\infty}^{-} \mid f \in l_{\infty}^{-}(G), f(e)=1\right\}>0,
$$

where $(\alpha(g) f)\left(g_{1}\right)=f\left(g_{1} g\right)$.

Proof. Let $G^{\infty}=G \times G \times \cdots$ be the space of trajectories of the random walk with the measure $\nu$ given by

$$
\nu\left(Z\left(g_{0}, \ldots, g_{n}\right)\right)=\delta_{g_{0}, e} \mu\left(g_{0}^{-1} g_{1}\right) \mu\left(g_{1}^{-1} g_{2}\right) \cdots \mu\left(g_{n-1}^{-1} g_{n}\right),
$$

where

$$
Z\left(g_{0}, \ldots, g_{n}\right)=\left\{\xi \in G^{\infty} \mid p_{k}(\xi)=g_{k}, 0 \leq k \leq n\right\} ;
$$

here $p_{k}$ is projection onto the $n$th component.

By the Shannon-McMillan-Breiman type theorem [11,7], there is a measurable set $\Omega \subset G^{\infty}$ with $\nu(\Omega)>1-\varepsilon$ and such that, for some $M$, if $n \geq M$ and $g \in p_{n}(\Omega)$ then

$$
\left|\frac{1}{n} \ln \nu\left(p_{n}^{-1}(g)\right)+h(G, \mu)\right|<\varepsilon
$$

or equivalently

$$
\left|\frac{1}{n} \ln \mu^{* n}(g)+h(G, \mu)\right|<\varepsilon .
$$

We define functions on $G$ by

$$
F_{k}(g)=\sum_{n \geq M} \nu\left(\Omega \cap p_{n}^{-1}(g) \cap p_{n+1}^{-1}(g k)\right)
$$

where $g \in G$ and $k \in K$.

Let $h=h(G, \mu)$ and assume $\delta=-h+2 \varepsilon<0$. We have

$$
F_{k}(g) \leq \sum_{n \geq M} e^{n \delta}<\infty .
$$


Note also that

$$
\begin{aligned}
\sum_{k \in K}\left(F_{k}(g)-F_{k}\left(g k^{-1}\right)\right)= & \sum_{n \geq M} \sum_{k \in K} \nu\left(\Omega \cap p_{n}^{-1}(g) \cap p_{n+1}^{-1}(g k)\right) \\
& -\sum_{n \geq M} \sum_{k \in K} \nu\left(\Omega \cap p_{n}^{-1}\left(g k^{-1}\right) \cap p_{n+1}^{-1}(g)\right) \\
= & \sum_{n \geq M} \nu\left(\Omega \cap p_{n}^{-1}(g)\right)-\sum_{n \geq M} \nu\left(\Omega \cap p_{n+1}^{-1}(g)\right) \\
= & \nu\left(\Omega \cap p_{M}^{-1}(g)\right) .
\end{aligned}
$$

On the other hand,

$$
\sum_{k \in K} F_{k}(g)=\sum_{n \geq M} \nu\left(\Omega \cap p_{n}^{-1}(g)\right) \leq \sum_{\substack{n \geq M \\ g \in p_{n}(\Omega)}} \mu^{* n}(g) .
$$

If $F=\sum_{k \in K} F_{k}$ and $F^{*}: \mathbb{N} \rightarrow \mathbb{R}$ is the decreasing rearrangement of $\{F(g) \mid$ $g \in G\}$, then it is easily seen that

$$
\begin{aligned}
F^{*}(1)+\cdots+F^{*}\left(\left[e^{n(h-\varepsilon)}\right]\right) & \leq \sum_{M \leq m \leq n} \sum_{g \in G} \mu^{* m}(g)+\sum_{k>n} e^{n(h-\varepsilon)} e^{-k(h-\varepsilon)} \\
& \leq n+\left(1-e^{-(h-\varepsilon)}\right)^{-1}
\end{aligned}
$$

This implies

$$
\begin{aligned}
\limsup _{q \rightarrow \infty} \frac{F^{*}(1)+\cdots+F^{*}(q)}{1+1 / 2+\cdots+1 / q} & \leq \limsup _{n \rightarrow \infty} \frac{F^{*}(1)+\cdots+F^{*}\left(\left[e^{n(h-\varepsilon)]}\right)\right.}{(n-1)(h-\varepsilon)} \\
& \leq \limsup _{n \rightarrow \infty} \frac{n+\left(1-e^{-(h-\varepsilon)}\right)^{-1}}{(n-1)(h-\varepsilon)}=(h-\varepsilon)^{-1},
\end{aligned}
$$

which further implies

$$
\sup _{g \in \mathbb{N}} \frac{F^{*}(1)+\cdots+F^{*}(q)}{1+1 / 2+\cdots+1 / q}=C<\infty .
$$

To conclude the proof we use duality as in [14]. Let $f \in l_{\infty}^{-}(G), f(e)=1$, and let $H_{k}=\alpha(k) f-f$ and $H_{k}^{*}$ be the decreasing rearrangements. We have

$$
\begin{aligned}
\left|\sum_{k \in K} \sum_{g \in G} H_{k}(g) F_{k}(g)\right| & \leq \sum_{k \in K} \sum_{j \in \mathbb{N}} H_{k}^{*}(j) F_{k}^{*}(j) \\
& \leq \sum_{k \in K} \sum_{j \in \mathbb{N}}\left(F_{k}^{*}(1)+\cdots+F_{k}^{*}(j)\right)\left(H_{k}^{*}(j)-H_{k}^{*}(j+1)\right) \\
& \leq \sum_{k \in K} \sum_{j \in \mathbb{N}} C(1+\cdots+1 / j)\left(H_{k}^{*}(j)-H_{k}^{*}(j+1)\right) \\
& \leq C \sum_{k \in K} \sum_{j \in \mathbb{N}} H_{k}^{*}(j) j^{-1} \leq|K| \cdot C \cdot \max _{k \in K}\left|H_{k}\right|_{\infty}^{-}
\end{aligned}
$$

The same type of argument shows that $\sum_{g \in G} F_{k}(g) f(g k), \sum_{g \in G} F_{k}(g) f(g)$ 
are absolutely convergent and hence

$$
\begin{aligned}
\sum_{k \in K} \sum_{g \in G} F_{k}(g) H_{k}(g) & =\sum_{k \in K} \sum_{g \in G} F_{k}(g)(f(g k)-f(g)) \\
& =\sum_{k \in K} \sum_{g \in G}\left(F_{k}\left(g k^{-1}\right)-F_{k}(g)\right) f(g) \\
& =-\sum_{g \in G} \nu\left(\Omega \cap p_{M}^{-1}(g)\right) f(g) .
\end{aligned}
$$

Since $\nu\left(\Omega \cap p_{M}^{-1}(g)\right)>0$ implies that $g \in K^{M}$, we have

$$
f(g) \geq 1-M \max _{k \in K}\left|H_{k}\right|_{\infty}^{-} \text {. }
$$

Hence if $\max _{k \in K}\left|H_{k}\right|_{\infty}<M^{-1}$ then

$$
\left|\sum_{k \in K} \sum_{g \in G} F_{k}(g) H_{k}(g)\right| \geq \nu(\Omega)\left(1-M \max _{k \in K}\left|H_{k}\right|_{\infty}^{-}\right),
$$

so that

$$
(1-\varepsilon)\left(1-M \max _{k \in K}\left|H_{k}\right|_{\infty}^{-}\right) \leq C|K| \max _{k \in K}\left|H_{k}\right|_{\infty}^{-},
$$

which implies a lower bound on $\max _{k \in K}\left|H_{k}\right|_{\infty}$. Q.E.D.

We have tried to make the preceding proof as self-contained as possible and have therefore avoided reference to the dual of $l_{\infty}^{-}$and to the duality arguments for quasicentral approximate units $[17,15]$ on which the last part of the proof was based. Though we will not go into the more refined questions concerning the relation to quasicentral approximate units here, we would like to point out that the preceding proof actually also yields a quantitatively more precise result. We will record here only its dual form, since this does not require further technical considerations.

The dual space $l_{1}^{+}(X)$ of $l_{\infty}^{-}(X)$ used implicitly in the proof of Theorem 1 consists of functions $F: X \rightarrow \mathbb{R}$ vanishing at $\infty$ such that

$$
|F|_{1}^{+}=\sup _{q \in \mathbb{N}} \frac{F^{*}(1)+\cdots+F^{*}(q)}{1+1 / 2+\cdots+1 / q} .
$$

The space $l_{1}^{+}(X)$ contains a closed subspace $l_{1}^{(0)+}(X)$, which is the closure of functions with finite support. The quotient space $l_{1}^{+} / l_{1}^{(0)+}$ will be denoted $l_{1}^{\sim+}(X)$ and we have the canonical surjection $\pi_{1}^{+}: l_{1}^{+} \rightarrow l_{1}^{\sim+}$ and the quotient norm ||$_{1}^{+}$. Then it can be shown that

$$
\left|\pi_{1}^{+}(F)\right|_{1}^{\sim^{+}}=\limsup _{q \rightarrow \infty} \frac{F^{*}(1)+\cdots+F^{*}(q)}{1+\cdots+1 / q} .
$$

The proof of Theorem 1 contains the following fact.

Corollary 2. Assume $h(G, \mu)>0$ and let $0<\varepsilon<\frac{1}{2} h(G, \mu)$. Then there are functions $F_{k} \in l_{1}^{+}(X) \quad(k \in K)$ such that:

(i) $F_{k} \geq 0$ and $\left|\sum_{k \in K} F_{k}\right|_{1}^{+} \leq(h(G, \mu)-\varepsilon)^{-1}$;

(ii) $\sum_{k \in K}\left(F_{k}-\alpha\left(k^{-1}\right) F_{k}\right) \geq 0$ and $\sum_{g \in G} \sum_{k \in k}\left(F_{k}-\alpha\left(k^{-1}\right) F_{k}\right)(g) \geq 1-\varepsilon$. 
This should be compared with the following, which is easily seen to be equivalent to Theorem 1 .

Corollary 3. If $h(G, \mu)>0$, then there are $F_{k} \in l_{1}^{+}(G) \quad(k \in K)$ such that

$$
\sum_{k \in K}\left(\alpha\left(k^{-1}\right) F_{k}-F_{k}\right)(g)=\delta_{e}(g) .
$$

Another equivalent form of Theorem 1 is obtained as follows. The condition that the inf appearing in the theorem be $>0$ is clearly equivalent to:

There is a constant $C>0$ such that for $f \in l_{\infty}^{-}(G)$ we have

$$
C \max _{g \in K}|\alpha(g) f-f|_{\infty}^{-} \geq|f(e)| \text {. }
$$

Moreover, since the left-hand side is invariant under left translations, this actually gives that the right-hand side can be replaced by $\|f\|_{\infty}$. Thus we have Corollary 4. If $h(G, \mu)>0$, there is a $C>0$ such that for $f \in l_{\infty}^{-}(G)$ we have

$$
C \max _{g \in K}|\alpha(g) f-f|_{\infty}^{-} \geq\|f\|_{\infty} .
$$

\section{Perturbation theory}

We shall now explain the connection with our work on perturbations of operators. Let $(\mathcal{J},|| \mathcal{J})$ be a normed ideal of compact operators [10] on a separable Hilbert space $\mathscr{H}$, and let $\tau=\left(T_{1}, \ldots, T_{n}\right)$ be an $n$-tuple of bounded operators on $\mathscr{H}$. We will assume throughout that the finite-rank operators are dense in $\mathscr{J}$. The invariant $k_{\mathscr{J}}(\tau)$ that we studied in [15-18] is defined by

$$
k_{\mathcal{J}}(\tau)=\liminf _{A \in \mathscr{R}_{1}^{+}}|[A, \tau]|_{\mathscr{J}}
$$

where $\mathscr{R}_{1}^{+}=\{A \in \mathscr{J}(\mathscr{H}) \mid 0 \leq A \leq I, A$ finite rank $\}$, the lim inf is with respect to the natural order on $\mathscr{R}_{1}^{+}$, and $|[A, \tau]|_{\mathscr{g}}$ stands for $\max _{1 \leq j \leq n} \mid\left[A, T_{j}\right]_{\mathscr{g}}$. The number $k_{\mathscr{J}}(\tau)$ can be viewed as a kind of "size $\mathscr{J}$ "-dimensional measure of $\tau$. In particular, $k_{\mathscr{f}}(\tau)>0$ is a statement of the type: $\tau$ has "size $\mathcal{J}$ "-dimensional measure $>0$. A very important role is played by the ideals $\mathscr{C}_{p}^{-}(1 \leq p \leq \infty)$, where

$$
|T|_{p}^{-}=\sum_{j \in \mathbb{N}} \lambda_{j} j^{-1+1 / p}
$$

with $\lambda_{1} \geq \lambda_{2} \geq \cdots$ the eigenvalues of $\left(T^{*} T\right)^{1 / 2}$. Dimension $p$ corresponds to the ideal $\mathscr{C}_{p}^{-}$. The ideal $\mathscr{C}_{\infty}^{-}$is the Macaev ideal and the corresponding number $k_{\mathscr{J}_{\infty}^{-}}$denoted $k_{\infty}^{-}$has remarkable properties $[15,17,18]$.

Note also that the Schatten-von Neumann classes $\mathscr{C}_{p}(1<p \leq \infty)$ are not the right type of ideals for these kind of questions since $k_{p}(\tau)$ for $p>1$ takes only the values 0 and $\infty[15,17]$.

Let $G$ be a discrete group with finite generating set $K=\left\{g_{1}, \ldots, g_{n}\right\}$, and let $\lambda$ be the regular representation of $G$ on $l^{2}(G)$. Then every normed ideal $\mathcal{J}$ defines a property of the group, namely

$$
k_{\mathscr{g}}\left(\lambda\left(g_{1}\right), \ldots, \lambda\left(g_{n}\right)\right)>0 \text {. }
$$

This property of the group does not depend on the choice of finite-generating set $K$. We have shown [15] that if $G=\mathbb{Z}^{n}$ then $0<k_{n}^{-}(\lambda(K))<\infty$, while 
$k_{p}^{-}(\lambda(K))=0$ if $p>n$. Similar results for the discrete Heisenberg group and $n=4$ were obtained by Bernier in [4]. If $G$ is a group which contains a free semigroup on two generators, we have shown [17] that $k_{\infty}^{-}(\lambda(K))>0$ and if $G$ has subexponential growth then $k_{\infty}^{-}(\lambda(K))=0$.

Given a normed ideal $\mathcal{J}$ we may consider the associated space

$$
l_{\mathscr{g}}(X)=\mathscr{J} \cap l^{\infty}(X)
$$

with $l^{\infty}(X)$ viewed as the algebra of multiplication operators in $l^{2}(X)$.

If $K \subset G$, we have shown [15] that

$$
k_{\mathscr{J}}(\lambda(K))=\liminf _{f \in \mathscr{R}_{1}^{+} \mathscr{F}(G)} \max _{k \in K}|\alpha(k) f-f|_{\mathscr{J}}
$$

where $\mathscr{F}(G)$ are the real functions on $G$ with finite support and

$$
\mathscr{R}_{1}^{+} \mathscr{F}(G)=\{f: G \rightarrow \mathbb{R} \mid \text { supp } f \text { finite }, 0 \leq f \leq 1\}
$$

is endowed with the natural order. In particular, $k_{\mathcal{g}}(\lambda(K))>0$ if and only if

$$
0<\inf \left\{\max _{k \in K}|\alpha(k) f-f|_{\mathscr{J}} \mid f \in l_{\mathscr{g}}(G), f(e)=1\right\} .
$$

By duality this is easily seen to be equivalent to the statement: there are $f_{k} \in l_{g}^{d}$ $(k \in K)$ such that

$$
\sum_{k \in K}\left(\alpha\left(k^{-1}\right) f_{k}-f_{k}\right)(g)=\delta_{e}(g)
$$

(Here $l_{g}^{d}$ is the dual of $l_{g}$. ) On the other hand, the considered property of the group is easily seen (as in the discussion preceding Corollary 4 ) to be equivalent to

Property $\mathscr{J}$. There is a constant $C>0$ such that if $f \in \mathscr{F}(G)$, then we have

$$
\|f\|_{\infty} \leq C \max _{k \in K}|\alpha(k) f-f|_{\mathcal{g}} .
$$

If $\mathcal{F}$ is the $p$-class, this condition is equivalent to a $p$-hyperbolicity condition in the sense of Yamasaki [19]. On the other hand, as pointed out above, except for $p=1$ when $\mathscr{C}_{1}^{-}=\mathscr{C}_{1}$, the good ideals are $\mathscr{C}_{p}^{-}$.

Thus Theorem 1 is actually about $k_{\infty}^{-}(\lambda(K))$ and we have

Corollary 5. If $h(G, \mu)>0$, then $k_{\infty}^{-}(\lambda(K))>0$.

By a result of [10] we know that $h(G, \mu)>0$ if $G$ is not amenable. Hence we also have

Corollary 6. If $G$ is not amenable, then $k_{\infty}^{-}(\lambda(K))>0$.

For dynamical systems our results [17] show that entropy and $k_{\infty}^{-}$are of the same order; it is therefore natural to ask if some converse to Theorem 1 holds for groups. 


\section{REFERENCES}

1. A. Avez, Entropie des groupes de type fini, C. R. Acad. Sci. Paris Sér. A 275 (1972), 1363-1366.

2. __ Théorème de Choquet-Deny pour les groupes à croissance non-exponentielle, $\mathrm{C} . \mathbf{R}$. Acad. Sci. Paris Sér. A 279 (1974), 25-28.

3. _ Harmonic functions on groups in differential geometry and relativity, Math. Phys. Appl. Math. 3 (1976), 27-32.

4. D. Bernier, Quasicentral approximate units for the discrete Heisenberg group, preprint, Berkeley, 1991.

5. D. Bisch, Entropy of groups and subfactors, preprint, UCLA, 1990.

6. A. Connes, Trace de Dixmier, modules de Fredholm et géométrie riemanniène, Nuclear Phys. B Proc. Suppl. 5 (1988), 65-70.

7. Y. Derrienic, Quelques applications du théorème ergodique sous-additif, Astérisque 74 (1980), 183-201.

8. E. B. Dynkin and M. B. Malyutov, Random walks on groups with a finite number of generators, Soviet Math. Dokl. 2 (1961), 399-402.

9. H. Furstenberg, Random walks on discrete subgroups of Lie groups, Adv. Probab. Related Topics, vol. 1, Dekker, New York, 1971, pp. 1-63.

10. I. C. Gohberg and M. G. Krein, Introduction to the theory of non-selfadjoint operators, Nauka, Moscow, 1965.

11. V. A. Khaimanovich and A. M. Vershik, Random walks on discrete groups: boundary and entropy, Ann. Probab. 3 (1983), 457-490.

12. V. A. Khaimanovich, Dirichlet norms, capacities and generalized isoperimetric inequalities for Markov operators, preprint.

13. S. Popa, Sous-facteurs, actions de groupes et cohomologie, C. R. Acad. Sci. Paris Sér. I Math. 309 (1989), 771-776.

14. N. T. Varopoulos, Long range estimates for Markov chains, Bull. Sci. Math. (2) 109 (1985), 225-252.

15. D. Voiculescu, Some results on norm-ideal perturbations of Hilbert space operators. I, J. Operator Theory 1 (1979), 3-37; II, J. Operator Theory 5 (1981), 77-100.

16. _ Hilbert space operators modulo normed ideals, Proc. Internat. Congr. Math., Warsaw, 1983, pp. 1041-1047.

17. $\ldots$, On the existence of quasicentral approximate units relative to normed ideals, Part I, J. Funct. Anal. 91 (1990), 1-36.

18. _ Entropy of dynamical systems and perturbations of operators. I, Ergodic Theory Dynamical Systems 11 (1991), 779-786; II, Houston Math. J. 17 (1991), 651-661.

19. M. Yamasaki, Parabolic and hyperbolic infinite networks, Hiroshima Math. J. (1977), 135-146.

Department of Mathematics, University of California, Berkeley, California 94720 